\title{
Contraceção em Adolescentes: Conhecimentos e Práticas em Portugal
}

\section{Contraception for Adolescents: Knowledge and Practices in Portugal}

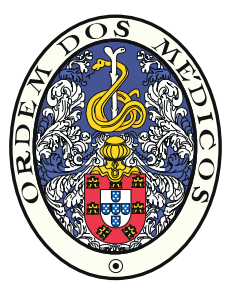

\author{
Patrícia MIRANDA ${ }^{1}$, Pascoal MOLEIRO ${ }^{1}$, Pedro GASPAR ${ }^{2}$, Alexandra LUZ ${ }^{1}$ \\ Acta Med Port 2019 Jul-Aug;32(7-8):505-513 - https://doi.org/10.20344/amp.11088
}

\section{RESUMO}

Introdução: Os adolescentes são um grupo de intervenção prioritária em saúde reprodutiva. Os médicos de medicina geral e familiar, ginecologistas e pediatras são os principais responsáveis pelo aconselhamento contracetivo.

Material e Métodos: Estudo transversal, correlacional, de natureza quantitativa, aprovado pela comissão de ética do Centro Hospitalar de Leiria. Dados colhidos em questionário online validado (alfa de Cronbach e Análise Fatorial Exploratória), constituído por itens de tipo diferencial semântico, com cinco pontos ("Discordo totalmente - 1" e "Concordo totalmente - 5"). Pontuações mais elevadas conotam-se com maior conhecimento ou adequabilidade na questão. Análise por género, especialidade, anos de carreira e grau de formação: especialista, interno de formação específica.

Resultados: Obtiveram-se 338 respostas (51\% médicos de medicina geral e familiar, $29 \%$ pediatras, $20 \%$ ginecologistas). Do total, $66 \%$ eram especialistas, $36 \%$ tinham menos de cinco anos de carreira e 17\% tinham mais de 20 anos de carreira. O conhecimento das recomendações foi superior entre os ginecologistas [4,38 (1,09); $p<0,001]$ e no género feminino $[3,65(1,41) ; p=0,039]$ com diferença estatisticamente significativa. Os ginecologistas receberam mais formação recentemente $[3,79(1,42)]$ e consideram estar atualizados $[4,41(0,70), p<0,001]$

Discussão: O questionário revelou validade e fiabilidade. Os ginecologistas estão mais familiarizados com a contraceção na adolescência, enquanto pediatras e clínicos mais novos referem maior necessidade de atualização. Os ginecologistas receberam formação recentemente e consideram estar atualizados, pelo que a formação na área parece ter impacto positivo.

Conclusão: O conhecimento acerca do aconselhamento contracetivo ainda não é ótimo, pelo que é fundamental investir na formação dos profissionais de saúde.

Palavras-chave: Aconselhamento; Adolescência; Contracepção; Contraceptivos; Portugal

\section{ABSTRACT}

Introduction: Adolescents are a target group for reproductive health intervention. Family physicians, gynecologists and pediatricians are the main providers of contraceptive counseling.

Material and Methods: Correlational, cross-sectional study, approved by the ethics committee of Leiria Hospital, Portugal. Data was collected through a validated online questionnaire (Cronbach's alpha and Exploratory Factorial Analysis) using a semantic differential scale (1 - Totally Agree; 5 - Totally Disagree). Higher scores were indicative of greater knowledge or suitability concerning the matter in question. Results were compared by gender, specialty, years of career and rank: consultant or resident.

Results: We received 338 responses (51\% family physicians, $29 \%$ pediatricians, $20 \%$ gynecologists), $66 \%$ were specialists, $36 \%$ had been working for less than 5 years and $17 \%$ for more than 20 years. Awareness of national recommendations was higher in gynecologists [4.38 (1.09)] and in the female gender [3.65 (1.41)] with statistically significant differences. Gynecologists had more recent training [3.79 (1.42)] and considered themselves to be up-to-date [4.41 (0.70)], $p<0.001$

Discussion: The questionnaire showed reliability and validity. Gynecologists were more familiarized with adolescent contraception; pediatricians and young clinicians highlighted the need for an update on this matter. Gynecologists had more training and considered themselves up-to-date, so training in this area seems to have a positive impact.

Conclusion: Knowledge about contraceptive counseling is not yet sufficient, so it is very important to invest in the continuous medical education of clinicians.

Keywords: Adolescent; Contraception; Contraceptive Agents; Counseling; Portugal

\section{INTRODUÇÃO}

Os adolescentes são considerados um grupo de intervenção prioritária na prevenção das infeções sexualmente transmissíveis (ISTs) e saúde reprodutiva. ${ }^{1}$

Portugal assistiu nas últimas décadas a um esforço na criação de consultas de atendimento a adolescentes e jovens, bem como consultas específicas de planeamento familiar, para melhorar a acessibilidade deste grupo etário aos recursos necessários para a (in)formação nesta matéria. Esta necessidade assume-se cada vez mais importante dada a diminuição na idade média da primeira relação sexual, que atualmente se cifra entre os 14 e os 15,6 anos. $^{2-6}$

A educação sexual e a promoção da saúde devem antecipar o início da vida sexual. ${ }^{7}$ As boas práticas nos cuidados antecipatórios ao adolescente e jovem incluem a elaboração da história sexual e reprodutiva, rastreio da gravidez e de ISTs, aconselhamento sobre métodos contracetivos e acessibilidade aos mesmos. ${ }^{3,8,9}$

Apesar da maioria dos/as adolescentes portugueses ser sexualmente ativa $(44 \%-95 \%),{ }^{2}$ o aconselhamento contracetivo junto de profissionais de saúde não é uma

\footnotetext{
1. Serviço de Pediatria. Hospital de Santo André. Centro Hospitalar de Leiria. Leiria. Portugal.

2. Center for Innovative Care and Health Technology. Instituto Politécnico de Leiria. Leiria. Portugal.

$\triangle$ Autor correspondente: Patrícia Miranda. pati_m16@hotmail.com

Recebido: 19 de julho de 2018 - Aceite: 06 de março de 2019 | Copyright @ Ordem dos Médicos 2019
} 
prática comum. ${ }^{2,4}$ A relação entre o aconselhamento contracetivo e a taxa de gravidez não desejada é inversa. ${ }^{10}$ Num estudo internacional, $14 \%$ dos abortos foram resultantes de um aconselhamento inadequado. ${ }^{11}$

O aconselhamento contracetivo realizado por profissionais de saúde está associado ao aumento do recurso à contraceção, ${ }^{12}$ e cada consulta deve ser uma oportunidade para rever o método utilizado e promover práticas adequadas no que respeita à sexualidade. ${ }^{13}$ A Academia Americana de Pediatria e a Sociedade Americana de Medicina do Adolescente recomendam uma consulta anual de planeamento familiar, no âmbito dos cuidados antecipatórios ao adolescente. ${ }^{13}$

De acordo com o descrito na literatura, o recurso à contraceção tem aumentado. ${ }^{3} \mathrm{O}$ preservativo é o método contracetivo de eleição na primeira relação sexual (RS) $(76 \%-96 \%)$ e nas subsequentes $(52 \%-69 \%) .{ }^{2}$ A disponibilização de novos métodos contracetivos, como o implante de progestativo, o contracetivo combinado transdérmico ou vaginal, e a extensão da recomendação de métodos já existentes à população adolescente (como o caso do dispositivo intrauterino, DIU) aumentaram o leque de escolha e permitem melhor adequação do método ao utilizador.

Para fornecer orientações dirigidas aos profissionais que lidam com esta temática na sua prática clínica, surgiram recomendações nacionais e internacionais específicas. ${ }^{8,14,15}$ Em 2012 foram publicadas as recomendações conjuntas da Sociedade Portuguesa de Medicina do Adolescente e da Sociedade Portuguesa de Contracepção sobre aconselhamento contracetivo na adolescência, um documento prático para ajudar todos os médicos a uniformizar a sua prática corrente. ${ }^{16}$ Atualmente em Portugal, dentro das especialidades médicas, os clínicos de Medicina Geral e Familiar, Ginecologia e Pediatria são os principais responsáveis pelo aconselhamento e prescrição contracetiva em adolescentes. Assume grande importância conhecer as práticas de aconselhamento e prescrição destas especialidades, e perceber como integram as recomendações mais atuais (nacionais e internacionais).

Deste modo, foram objetivos deste estudo, avaliar os conhecimentos e práticas destes clínicos relativos à contraceção na adolescência e comparar os resultados entre as diferentes especialidades, graus de formação e anos de carreira.

\section{MATERIAL E MÉTODOS}

Estudo transversal e correlacional, baseado em questionário de autopreenchimento, disponibilizado online, entre setembro e dezembro de 2016 , divulgado pelas Sociedade Portuguesa de Pediatria (http://www.spp.pt/), Sociedade Portuguesa de Ginecologia (http://www.spginecologia.pt/) e Associação Portuguesa de Medicina Geral e Familiar (http://www.apmgf.pt/).

A fidelidade das questões/itens averiguou-se pelo coeficiente de consistência interna (alfa de Cronbach) e a validade averiguou-se pela correlação de cada item com a escala total excluindo o item e ainda pela análise fatorial exploratória (AFE) pelo método Principal Axis Factoring, seguida de rotação ortogonal do tipo Varimax.

Mantiveram-se os itens que se apresentassem, pela correlação de cada item com a escala total excluindo o item, próximos de 0,2 , ou cuja remoção não aumentasse significativamente a fidelidade do conjunto de itens, e também os itens que saturassem apenas num fator com peso fatorial próximo ou superior a $0,30 .{ }^{17}$ Foi verificada a adequabilidade da análise fatorial pelo teste Kaiser-Meyer-Olkin (KMO) e com o teste de esfericidade de Bartlett verificou-se que a matriz de correlação da população não é uma matriz identidade. ${ }^{18}$

O critério utilizado para a extração de fatores incidiu na análise do scree-plot, ${ }^{19}$ representação gráfica dos fatores no eixo das abcissas e dos respetivos valores próprios no eixo das ordenadas. Retiveram-se os fatores até àquele em que se observou o ponto de inflexão da curva que relaciona o número do fator e o respetivo eigenvalue.

Foram seguidas as orientações para identificação de pesos fatoriais significantes com base na dimensão da amostra. ${ }^{17}$ Para um nível de significância de 0,05 e amostra próxima de 350 indivíduos considerou-se adequado um peso fatorial próximo ou superior a 0,30.

A variância comum explicada, que deverá ser superior a $50 \%{ }^{20}$ verificou-se na Escala de Aconselhamento Contracetivo na Adolescência - Práticas [EACA-P (61, 80\%)], mas ficou abaixo no caso da Escala de Aconselhamento Contracetivo na Adolescência - Conhecimentos [EACA-C $(43,47 \%)]$. Contudo, pela sua pertinência teórica, optou-se por não excluir as variáveis com cargas fatoriais relativamente baixas em todos os fatores, para melhorar a solução fatorial geral e aumentar a percentagem da variância comum explicada nesta escala.

A amostra por cotas de 338 clínicos foi analisada por género [masculino $(M)$; feminino $(F)$ ], grupo etário [G1 - entre os 25 e os 34 anos; G2 - dos 35 aos 44 anos; G3 - dos 45 aos 54 anos e G4 - 55 ou mais anos], especialidade [Ginecologia (G); Pediatria (P); Medicina Geral e Familiar (MGF)], formação [Especialista (E); Interno de Formação Específica (IFE)] e anos de carreira: [A1 (< 5 anos); A2 (5 - 10 anos); A3 (11 - 20 anos) e A4 (> 20 anos)].

A análise estatística foi efetuada em SPSS 24®, tendose fixado um nível de significância de 0,05.

Foram observados os pressupostos do teorema do limite central, tendo-se usado o teste $t$ de Student em amostras independentes, para comparações em função do género e graus de formação, e ANOVA, com teste post hoc de Tukey, para comparações em função do grupo etário, especialidade e anos de carreira.

O estudo foi aprovado pela Comissão de Ética do Centro Hospitalar de Leiria. Foi assegurada participação voluntária, anónima e confidencial.

\section{RESULTADOS}

Obtiveram-se 338 respostas [51\% (172) MGF, 29\% (98) P, 20\% (68) G], sendo 66\% (223) E e 84\% (284) F. Mais de metade dos respondentes pertencia à faixa etária dos 25 
aos 34 anos (58\%, 196), 23\% (78) dos 35 aos 44 anos, $7 \%$ (24) dos 45 aos 54 anos e 12\% (40) tinha 55 anos ou mais. Tinha menos de cinco anos de carreira 36\% (122), 35\% (118) tinha 5 a 10 anos de carreira, $12 \%$ (40) tinha 11 a 20 anos de carreira e $17 \%$ (58) tinha pelo menos 20 anos de carreira.

Para a construção e validação da EACA-C reunimos 20 questões emergentes da literatura consultada e das recomendações nacionais e internacionais específicas, solicitando-se depois a peritos da área a avaliação da pertinência e adequação das questões, que em rondas de analise e correção sucessivas, por parte dos investigadores, originaram itens do tipo diferencial semântico, com cinco pontos, em que "Discordo totalmente - 1" e "Concordo totalmente - 5".

A maioria dos itens apresentou correlações com a escala total excluindo o item superiores a 0,2 e contribuiu para alfa de Cronbach. Os valores do alfa de Cronbach foram de 0,820 a 0,846 para os itens e 0,846 para o total da escala (Tabela 1).

Tabela 1 - Estatísticas de homogeneidade dos itens, coeficientes de consistência interna e análise fatorial exploratória da EACA-C

\begin{tabular}{|c|c|c|c|c|c|c|c|c|c|}
\hline Descrição do item & Limites & M & DP & $\begin{array}{c}r \text { do } \\
\text { total } \\
\text { sem o } \\
\text { item }\end{array}$ & $\begin{array}{c}\text { a de } \\
\text { Cronbach } \\
\text { quando } \\
\text { o item é } \\
\text { excluído }\end{array}$ & $h^{2}$ & F1 & F2 & F3 \\
\hline $\begin{array}{l}\text { 7a. Conheço a existência dos critérios para o } \\
\text { uso de contracetivos da Centers for Disease }\end{array}$ & $1-5$ & 3,23 & 0,47 & 0,46 & 0,831 & 0,60 & 0,755 & $-0,040$ & $-0,166$ \\
\hline
\end{tabular}

Control and Prevention (2010)

(1)

0,831

7b. Conheço as recomendações para o aconselhamento contracetivo divulgadas pela Sociedade Portuguesa de Medicina do Adolescente e pela Sociedade Portuguesa da $1-5 \quad 3,58 \quad 1,43 \quad 0,60$ $0,55 \quad \mathbf{0 , 7 2 7} \quad 0,118$

0,093 Contracepção (2014)

7c. Conheço a existência dos critérios para o uso de contracetivos da Organização Mundial da Saúde (2015)$$
1-
$$

$1-5$

3,5

1,34

0,57

0,826

0,53

0,715

0,140

$-0,043$

7k. Estou familiarizado com o conceito

da "Dupla Proteção" aplicável no aconselhamento contracetivo dos $1-$

$1-5 \quad 4,63 \quad 0,81$

0,40

0,835

0,26

0,419

0,093

0,274

adolescentes

8c. Quando prescrevo uma pílula combinada, adequo a dose de estrogénio à adolescente em causa

8e. Quando prescrevo uma pílula combinada, adequo o tipo de progestativo à adolescente em causa

9a. Considero que os meus conhecimentos estão atualizados relativamente ao aconselhamento contracetivo na adolescência

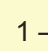

$1-5 \quad 4,35 \quad 0,91$

0,831

0,38

0,564

0,244

0,057

$\begin{array}{lllllllll}1-5 & 4,11 & 1,06 & 0,53 & 0,829 & 0,43 & \mathbf{0 , 6 3 3} & 0,146 & 0,105 \\ 1-5 & 3,57 & 1,10 & 0,741 & 0,820 & 0,67 & \mathbf{0 , 7 5 0} & 0,281 & 0,179 \\ 1-5 & 3,02 & 1,52 & 0,54 & 0,827 & 0,51 & \mathbf{0 , 7 0 7} & 0,052 & 0,075\end{array}$
formação direta sobre aconselhamento contracetivo na adolescência (ex. reuniões científicas, cursos, ações de formação)

$9 \mathrm{c}$. Nos últimos 3 anos recebi alguma formação indireta sobre aconselhamento contracetivo na adolescência (ex. revistas médicas, pesquisa online)

8b. Os métodos barreira são os únicos que protegem contra as infeções sexualmente transmissíveis, desde que utilizados corretamente

$8 \mathrm{~g}$. O aconselhamento sobre o anel vaginal faz parte da minha abordagem aos métodos contracetivos na adolescência

$$
1-5
$$

$1-5$ $3,69 \quad 1,25$$$
1-
$$

0,68

0,821

0,64

0,726

0,104

0,323

8h. O aconselhamento sobre o sistema transdérmico ("selo") faz parte da minha abordagem aos métodos contracetivos na adolescência$$
1-
$$

$3,28 \quad 1,29$

0,47

0,831

0,54

0,372

0,62

$-0,120$

8i. Considero o implante contracetivo um método de $1^{\mathrm{a}}$ linha na contraceção em adolescentes
0,20

0,844

0,25

$-0,007$

0,497

0,079
$1-5 \quad 2,86 \quad 1,28$ 
Tabela 1 (cont.) - Estatísticas de homogeneidade dos itens, coeficientes de consistência interna e análise fatorial exploratória da EACA-C

\begin{tabular}{|c|c|c|c|c|c|c|c|c|c|}
\hline Descrição do item & Limites & M & DP & $\begin{array}{l}r \text { do } \\
\text { total } \\
\text { sem o } \\
\text { item }\end{array}$ & $\begin{array}{c}\alpha \text { de } \\
\text { Cronbach } \\
\text { quando } \\
\text { o item é } \\
\text { excluído }\end{array}$ & $h^{2}$ & F1 & F2 & F3 \\
\hline $\begin{array}{l}\text { 8j. O dispositivo intrauterino é um método } \\
\text { indicado na adolescência, em determinados } \\
\text { contextos }\end{array}$ & $1-5$ & 3,31 & 1,29 & 0,46 & 0,831 & 0,41 & 0,266 & 0,548 & 0,207 \\
\hline $\begin{array}{l}\text { 8k. Não existem contraindicações ao uso da } \\
\text { contraceção de emergência }\end{array}$ & $1-5$ & 2,52 & 1,47 & 0,35 & 0,838 & 0,20 & 0,269 & 0,318 & 0,163 \\
\hline $\begin{array}{l}\text { 71. A contraceção pode ser iniciada no dia } \\
\text { da consulta }\end{array}$ & $1-5$ & 4,22 & 1,11 & 0,40 & 0,834 & 0,48 & 0,160 & 0,344 & 0,581 \\
\hline $\begin{array}{l}i 7 \mathrm{~m} \text {. A contraceção apenas pode ser iniciada } \\
\text { no } 1^{\circ} \text { dia da menstruação }\end{array}$ & $1-5$ & 4,35 & 1,04 & 0,23 & 0,841 & 0,49 & $-0,077$ & 0,328 & 0,614 \\
\hline $\begin{array}{l}\text { i 8a. Os métodos naturais são uma das } \\
\text { primeiras opções de aconselhamento } \\
\text { contracetivo nos adolescentes saudáveis }\end{array}$ & $1-5$ & 4,74 & 0,80 & 0,20 & 0,841 & 0,33 & 0,152 & $-0,111$ & 0,543 \\
\hline $\begin{array}{l}i \text { 8f. Nas adolescentes, prescrevo a pílula só } \\
\text { com progestativo como } 1^{\mathrm{a}} \text { linha }\end{array}$ & $1-5$ & 4,35 & 0,91 & 0,21 & 0,841 & 0,47 & 0,135 & $-0,145$ & 0,657 \\
\hline $\begin{array}{l}i \text { 8l. A contraceção de emergência requer } \\
\text { sempre prescrição médica }\end{array}$ & $1-5$ & 3,84 & 1,41 & 0,18 & 0,846 & 0,25 & $-0,038$ & 0,248 & 0,438 \\
\hline TOTAL EACA-C & & & & & 0,846 & & & & \\
\hline Eigenvalues & & & & & & & 5,53 & 1,86 & 1,31 \\
\hline Variância Explicada $(\Sigma=43,47 \%)$ & & & & & & & 27,66 & 9,28 & 6,44 \\
\hline Número de itens & & & & & & & 9 & 6 & 5 \\
\hline Alfa de Cronbach do fator & & & & & & & 0,882 & 0,599 & 0,557 \\
\hline $\begin{array}{l}\text { KMO }=0,847 ; \text { Teste de esferacidade } \\
\text { de Bartlett }=1992,67, p<0,0001\end{array}$ & & & & & & & & & \\
\hline
\end{tabular}

M: média; DP: desvio padrão; $i$ : item invertido

A análise fatorial exploratória (AFE) pelo método Principal Axis Factoring, seguida de rotação ortogonal do tipo Varimax, após avaliação de várias soluções, conduziu a uma solução de três fatores que se revelou a mais apropriada quer pelas características psicométricas quer pela validade facial. A maioria dos itens saturou apenas num fator e apresentou saturação, no fator correspondente, superior a 0,30 pontos. ${ }^{17}$ Nos casos em que se observou saturação de um item em mais do que um fator, optou-se pela sua inclusão no fator em que o saturou mais, respeitando uma diferença igual ou superior a 0,1 pontos (Tabela 1).

As correlações entre os três fatores e o total da escala EACA-C são positivas e muito significativas $(p<0,01)$ variando de moderadas (fator2 - 0,558) a muito fortes (fator1 - 0,911).

Para a construção e validação da EACA-P reunimos um conjunto de 15 questões emergentes da literatura consultada e das recomendações nacionais e internacionais específicas, solicitando-se depois a peritos da área a avaliação da pertinência e adequação das questões, que foram submetidas a rondas de análise e correção sucessivas, por parte dos investigadores, de forma a redigirem-se itens da escala do tipo diferencial semântico, com cinco pontos, em que "Discordo totalmente - 1" e "Concordo totalmente - 5".

A maioria dos itens apresentou correlações com a escala total excluindo o item superiores a 0,2 e contribui para alfa de Cronbach. Optámos por manter as exceções pela pertinência teórica e porque ao serem removidos não aumentam de forma substancial a fiabilidade. Os valores do alfa de Cronbach são de 0,794 a 0,728 para os itens e 0,764 para o total da escala, e na esmagadora maioria dos casos, os valores descem quando os itens são excluídos, ou seja, a sua presença melhora a homogeneidade da escala (Tabela 2).

AAFE pelo método Principal Axis Factoring, seguida de rotação ortogonal do tipo Varimax, após avaliação de várias soluções, conduziu a uma solução de três fatores que se revelou a mais apropriada quer pelas características psicométricas quer pela validade facial. Também nesta escala a maioria dos itens saturou apenas num fator e apresentou saturação, no fator correspondente, superior a 0,30 pontos $^{17}$ e nos casos em que se observou saturação de um item em mais do que um fator, optou-se pela sua inclusão no fator em que o saturou mais, respeitando uma diferença igual ou superior a 0,1 pontos (Tabela 2 ).

As correlações entre os três fatores e o total da escala EACA-P foram todas positivas e muito significativas $(p<0,01)$ variando de moderadas (fator $3-0,415)$ a muito fortes (fator $1-0,878$ ).

Nas tabelas dos resultados encontram-se destacados os itens onde se observaram diferenças estatisticamente significativas. 
Tabela 2 - Estatísticas de homogeneidade dos itens, coeficientes de consistência interna e análise fatorial exploratória da EACA-P

\begin{tabular}{|c|c|c|c|c|c|c|c|c|c|}
\hline Descrição do item & Limites & M & DP & $\begin{array}{l}r \text { do } \\
\text { total } \\
\text { sem o } \\
\text { item }\end{array}$ & $\begin{array}{c}\alpha \text { de } \\
\text { Cronbach } \\
\text { quando } \\
\text { o item é } \\
\text { excluído }\end{array}$ & $\mathbf{h}^{2}$ & F1 & F2 & F3 \\
\hline $\begin{array}{l}\text { 6b. Asseguro o direito à confidencialidade } \\
\text { e à privacidade do jovem, nomeadamente } \\
\text { atendimento individualizado }\end{array}$ & $1-5$ & 4,61 & 0,70 & 0,46 & 0,747 & 0,37 & 0,602 & $-0,001$ & 0,090 \\
\hline $\begin{array}{l}\text { 6c. Abordo o aconselhamento contracetivo } \\
\text { no contexto de uma abordagem integrada da } \\
\text { sexualidade, de acordo com o grupo etário, } \\
\text { género e risco individual }\end{array}$ & $1-5$ & 4,49 & 0,79 & 0,67 & 0,729 & 0,75 & 0,836 & $-0,077$ & 0,220 \\
\hline $\begin{array}{l}\text { 6d. Sinto-me à vontade para abordar o } \\
\text { aconselhamento contracetivo }\end{array}$ & $1-5$ & 4,38 & 0,92 & 0,61 & 0,730 & 0,64 & 0,802 & $-0,008$ & 0,009 \\
\hline $\begin{array}{l}\text { 6e. Abordo as infeções sexualmente } \\
\text { transmissíveis }\end{array}$ & $1-5$ & 4,46 & 0,80 & 0,60 & 0,735 & 0,65 & 0,795 & $-0,091$ & 0,109 \\
\hline $\begin{array}{l}\text { 6f. Abordo os afetos e a sexualidade no } \\
\text { contexto de uma abordagem integrada da } \\
\text { sexualidade, de acordo com o grupo etário, } \\
\text { género e risco individual }\end{array}$ & $1-5$ & 4,04 & 0,97 & 0,46 & 0,743 & 0,45 & 0,616 & $-0,113$ & 0,235 \\
\hline $\begin{array}{l}\text { 6g. No âmbito do aconselhamento na } \\
\text { adolescência, faz parte da minha prática a } \\
\text { abordagem de todos os métodos contracetivos }\end{array}$ & $1-5$ & 4,08 & 1,07 & 0,59 & 0,729 & 0,70 & 0,832 & $-0,063$ & $-0,048$ \\
\hline $\begin{array}{l}\text { 6h. Na minha prática médica diária, } \\
\text { procuro adequar o método contracetivo ao } \\
\text { adolescente }\end{array}$ & $1-5$ & 4,50 & 0,85 & 0,66 & 0,728 & 0,72 & 0,848 & 0,024 & 0,003 \\
\hline $\begin{array}{l}\text { 6i. Na minha prática médica diária, procuro } \\
\text { respeitar a preferência do adolescente pelo }\end{array}$ & $1-5$ & 4,50 & 0,80 & 0,64 & 0,731 & 0,66 & 0,810 & 0,045 & 0,038 \\
\hline
\end{tabular}
método contracetivo

7n. Na minha prática clínica, aproveito para abordar a contraceção e rever o método contracetivo utilizado, sempre que me é solicitada a contraceção de emergência

i 7d. Na minha prática clínica tenho como pressuposto a idade como uma limitação à prescrição dos métodos contracetivos

i $7 \mathrm{~g}$. Antes de prescrever qualquer método contracetivo, efetuo sistematicamente avaliação analítica

$i 7 \mathrm{~h}$. Antes de prescrever qualquer método contracetivo, efetuo sistematicamente o exame ginecológico à adolescente

i 7i. Antes de prescrever qualquer método contracetivo, efetuo sistematicamente a citologia cervical à adolescente

7e. Antes de prescrever qualquer método contracetivo, efetuo sistematicamente a avaliação da tensão arterial

$\begin{array}{llllllll}1-5 & 4,53 & 0,91 & 0,50 & 0,740 & 0,40 & \mathbf{0 , 5 6 7} & 0,081\end{array}$

0,262

7f. Antes de prescrever qualquer método contracetivo, efetuo sistematicamente a determinação do peso e do índice de massa corporal

TOTAL EACA-P

\section{Eigenvalues}

Variância Explicada $(\Sigma=61,80 \%)$

$\begin{array}{llllllllll}1-5 & 3,69 & 1,20 & 0,11 & 0,779 & 0,21 & 0,116 & \mathbf{0 , 3 9 1} & -0,207 \\ 1-5 & 3,74 & 1,20 & 0,08 & 0,783 & 0,65 & -0,014 & \mathbf{0 , 7 6 8} & -0,239 \\ 1-5 & 3,71 & 1,34 & 0,03 & 0,794 & 0,72 & -0,172 & \mathbf{0 , 8 2 4} & 0,092 \\ & & & & & & & & \\ 1-5 & 4,32 & 1,00 & 0,16 & 0,770 & 0,70 & -0,051 & \mathbf{0 , 8 3 1} & 0,077\end{array}$

$\begin{array}{lllllllll}1-5 & 4,29 & 1,01 & 0,25 & 0,762 & 0,82 & 0,171 & -0,110 & \mathbf{0 , 8 8 3}\end{array}$

Número de itens

Alfa de Cronbach do factor

$1-5 \quad 4,42 \quad 0,88$

0,30

0,757

0,83

$0,206 \quad-0,089$

0,884

$\mathrm{KMO}=0,859$; Teste de esferacidade

de Bartlett $=2442,13, p<0,0001$

M: média; DP: desvio padrão; $i$ : item invertido 
Observa-se na Tabela 3 que os clínicos do género feminino estão mais familiarizados com a temática da contraceção na adolescência. Destaca-se que estes clínicos revelaram maior conhecimento quer das recomendações nacionais (7b) [F 3,65 (1,41); M 3,22 (1,49), $p=0,039]$, quer do conceito de dupla proteção (7k) [F 4,69 $(0,75)$; M 4,35 (1,02), $p=0,004]$. Na prática clínica, são igualmente os clínicos do género feminino aqueles que, com maior frequência, adequam a dose de estrogénio de uma pílula combinada à adolescente $(8 \mathrm{c})[\mathrm{F} 4,43(0,86)$; M 3,95 $(1,06)$, $p<0,001$ ] e revêm o método contracetivo utilizado, sempre que é solicitada a contraceção de emergência (7n) [F 4,58 $(0,88) ;$ M 4,31 (1,03), $p=0,046]$.

Em função do grau de formação, na mesma tabela, há a destacar que os IFEs abordam o anel vaginal com maior frequência (8g) [E 3,63 (1,45); IFE 3,92 $(1,13), p=0,042]$ e, por outro lado, os $E$ abordam mais frequentemente as ISTs (6e) [E 4,52 (0,80); IFE 4,34 (0,78), $p=0,04]$.

No Apêndice 1 constata-se que os clínicos com pelo menos 55 anos (G4) estão mais familiarizados com as recomendações nacionais (7b) [25 - 34 anos: $3,71(1,38)$; 35 - 44 anos: $3,16(1,52)$; $45-54$ anos: 3,67 $(1,43)$; $\geq 55$ anos: $3,73(1,41), p=0,04]$. Foram igualmente os mais velhos (G4) aqueles que consideraram estar mais à vontade para abordar esta temática (6d) [25 - 34 anos: 4,40 $(0,86)$; $35-44$ anos: 4,27 (1,00); $45-54$ anos: 4,04 (1,27); $\geq 55$ anos: $4,68(0,69), p=0,003]$. Já a abordagem dos métodos contracetivos mais recentes, como o anel vaginal (8g), está mais frequentemente integrada na abordagem contracetiva dos clínicos mais novos [25 - 34 anos: 3,94 $(1,20)$; $35-44$ anos: $3,60(1,44) ; 45-54$ anos: 2,92 (1,64); $\geq 55$ anos: $3,46(1,52), p=0,001]$ e com menos anos de

Tabela 3 - Resultados em função do género e do grau de formação (teste $t$ para amostras independentes)

\begin{tabular}{|c|c|c|c|c|}
\hline \multicolumn{5}{|l|}{ RESULTADOS EM FUNÇÃO DO GÉNERO } \\
\hline Questão & M & $\mathbf{F}$ & $t$ & $p$ \\
\hline $\begin{array}{l}\text { 6b. Asseguro o direito à confidencialidade e à privacidade do jovem, } \\
\text { nomeadamente atendimento individualizado }\end{array}$ & $4,62(0,65)$ & $4,61(0,71)$ & 0,101 & 0,920 \\
\hline $\begin{array}{l}\text { 7b. Conheço as recomendações para o aconselhamento contracetivo } \\
\text { divulgadas pela Sociedade Portuguesa de Medicina do Adolescente e pela } \\
\text { Sociedade Portuguesa da Contracepção (2014) }\end{array}$ & $3,22(1,49)$ & $3,65(1,41)$ & $-2,072$ & 0,039 \\
\hline $\begin{array}{l}\text { 7k. Estou familiarizado com o conceito da "Dupla Proteção" aplicável no } \\
\text { aconselhamento contracetivo dos adolescentes }\end{array}$ & $4,35(1,02)$ & $4,69(0,75)$ & $-2,928$ & 0,004 \\
\hline $\begin{array}{l}\text { 8c. Quando prescrevo uma pílula combinada, adequo a dose de estrogénio } \\
\text { à adolescente em causa }\end{array}$ & $3,95(1,06)$ & $4,43(0,86)$ & $-3,663$ & $<0,001$ \\
\hline $\begin{array}{l}\text { 8e. Quando prescrevo uma pílula combinada, adequo o tipo de progestativo } \\
\text { à adolescente em causa }\end{array}$ & $3,65(1,25)$ & $4,20(0,99)$ & $-3,576$ & $<0,001$ \\
\hline $\begin{array}{l}\text { i 8a. Os métodos naturais são uma das primeiras opções de } \\
\text { aconselhamento contracetivo nos adolescentes saudáveis }\end{array}$ & $4,55(1,05)$ & $4,78(0,74)$ & $-1,979$ & 0,049 \\
\hline$i$ 8f. Nas adolescentes, prescrevo a pílula só com progestativo como $1^{\mathrm{a}}$ linha & $4,11(1,12)$ & $4,40(0,85)$ & $-2,184$ & 0,030 \\
\hline $\begin{array}{l}\text { 8b. Os métodos barreira são os únicos que protegem contra as infeções } \\
\text { sexualmente transmissíveis, desde que utilizados corretamente }\end{array}$ & $4,64(0,87)$ & $4,87(0,57)$ & $-2,513$ & 0,012 \\
\hline $\begin{array}{l}\text { 8i. Considero o implante contracetivo um método de } 1^{\text {a }} \text { linha na contraceção } \\
\text { em adolescentes }\end{array}$ & $2,55(1,34)$ & $2,92(1,26)$ & $-2,013$ & 0,045 \\
\hline $\begin{array}{l}\text { 7n. Na minha prática clínica, aproveito para abordar a contraceção e rever } \\
\text { o método contracetivo utilizado, sempre que me é solicitada a contraceção }\end{array}$ & $4,31(1,03)$ & $4,58(0,88)$ & $-2,002$ & 0,046 \\
\hline
\end{tabular}
de emergência

\section{RESULTADOS EM FUNÇÃO DO GRAU DE FORMAÇÃO}

IFE

$t$

\begin{tabular}{|c|c|c|c|}
\hline $4,65(0,68)$ & $4,53(0,73)$ & $-1,561$ & 0,120 \\
\hline $3,63(1,45)$ & $3,92(1,13)$ & 39,413 & 0,042 \\
\hline $3,19(1,33)$ & $3,53(1,18)$ & 21,822 & 0,015 \\
\hline $4,52(0,80)$ & $4,34(0,78)$ & $-2,068$ & 0,040 \\
\hline $4,14(0,95)$ & $3,84(0,99)$ & $-2,760$ & 0,006 \\
\hline $4,43(0,91)$ & $4,11(1,12)$ & $-2,621$ & 0,009 \\
\hline $4,39(1,00)$ & $4,11(1,00)$ & $-2,396$ & 0,017 \\
\hline
\end{tabular}

6b. Asseguro o direito à confidencialidade e à privacidade do jovem, nomeadamente atendimento individualizado

8g. O aconselhamento sobre o anel vaginal faz parte da minha abordagem aos métodos contracetivos na adolescência

8j. O dispositivo intrauterino é um método indicado na adolescência, em determinados contextos

6e. Abordo as infeções sexualmente transmissíveis

6f. Abordo os afetos e a sexualidade no contexto de uma abordagem integrada da sexualidade, de acordo com o grupo etário, género e risco individual

$i$ ii. Antes de prescrever qualquer método contracetivo, efetuo sistematicamente a citologia cervical à adolescente

7 e. Antes de prescrever qualquer método contracetivo, efetuo sistematicamente a avaliação da tensão arterial

E: especialista; IFE: interno de Formação Específica; M: masculino; F: feminino 
carreira [< 5 anos: 3,89 (1,20); $5-10$ anos: 3,85 (1,29); $11-20$ anos: $3,55(1,48)$; $\geq 20$ anos: $3,28(1,59), p=0,019]$.

Relativamente à especialidade, globalmente os $\mathrm{G}$ têm maior nível de conhecimento no que respeita às recomendações nacionais (7b) [G 4,38 (1,09); P 3,36 (1,50); MGF $3,40(1,41), p<0,001]$, ao conceito de dupla proteção $(7 \mathrm{k})$ [G 4,95 (0,21); P 4,43 (1,03); MGF 4,62 (0,76), $p<0,001]$, e mais frequentemente consideram estar à vontade para abordar o aconselhamento contracetivo (6d) [G 4,85 $(0,36)$; P 3,71 (1,12); MGF 4,58 $(0,71), p<0,001]$.

Os $\mathrm{G}$ abordam com maior frequência o anel vaginal $(8 \mathrm{~g})$ [G 4,36 (1,02); P 2,84 (1,43); MGF 4,00 (1,17), $p<0,001$ ], o sistema transdérmico (8h) [G 4,24 (0,98); P 2,78 $(1,29)$; MGF 3,20 (1,20), $p<0,001]$, as ISTs (6e) [G 4,65 $(0,57)$; P 4,05 (1,06); MGF 4,62 $(0,58), p<0,001]$ e revêm o método quando é solicitada a contraceção de emergência (7n) [G 4,76 (0,61); MGF 4,75 (0,52); P 4,00 (1,31), $p<0,001$ ].

$\mathrm{Na}$ prescrição de um contracetivo combinado, os $\mathrm{G}$ adequam mais frequentemente a dose de estrogénio à adolescente (8c) [G 4,61 (0,72); P 4,09 (1,14); MGF 4,40 $(0,78), p=0,001]$ e o tipo de progestativo (8e) [G 4,71 $(0,58) ; P 3,87(1,20) ;$ MGF 4,02 (1,03), $p<0,001]$. Já os MGF consideram mais frequentemente o implante contracetivo como método de primeira linha (8i) [G 2,58 $(1,23)$; P 2,33 (1,24); MGF 3,27 (1,18), $p<0,001$ ] e avaliam de forma mais sistemática os parâmetros antropométricos antes da prescrição contracetiva (7f) [G 4,18 $(0,99)$; P 4,39 $(0,96)$; MGF 4,53 (0,76), $p=0,020]$.

Foram também os $\mathrm{G}$ aqueles que com maior frequência referiram respeitar a confidencialidade e a privacidade do jovem aquando do aconselhamento contracetivo [G 4,77 $(0,49)$; MGF 4,65 (0,11); P 4,43 (0,91), $p=0,005]$.

Em relação à perceção da formação os G e G4 ( $\geq 55$ anos) receberam mais formação direta recentemente (9b): G 3,79 (1,42); MGF 2,89 (1,47); P 2,73 (1,52), $p<0,001$ e 25 - 34 anos: $3,14(1,49) ; 35-44$ anos: $2,56(1,44) ; 45-54$ anos: $3,04(1,71) ; \geq 55$ anos: $3,29(1,60), p=0,022$, sendo igualmente os grupos que consideram estar mais atualizados (9a): G 4,41 (0,70); MGF 3,64 (0,90); P 2,88 $(1,21)$, $p<0,001$ e $25-34$ anos: $3,59(1,01) ; 35-44$ anos: 3,31 $(1,18) ; 45-54$ anos: $3,50(1,25) ; \geq 55$ anos: $3,95(1,18)$, $p=0,024$. Por outro lado, os $\mathrm{P}$, os IFE e os médicos com menos de cinco anos de carreira (A1) são os que consideram mais pertinente a formação na área $[P 4,54(0,79)$; MGF 4,45 (0,80); G 4,14 (1,04), $p=0,010$ ], [IFE 4,57 $(0,75)$; E 4,33 (0,89), $p=0,009],[<5$ anos: $4,67(0,66) ; 5-10$ anos: 4,37 (0,80); $11-20$ anos: 4,20 (0,85); $\geq 20$ anos: 4,09 $(1,13), p<0,001$ ] na resposta à questão isolada "Considero pertinente a realização de formação adicional nesta área para a minha prática clínica".

\section{DISCUSSÃO}

A educação sexual e a promoção da saúde devem antecipar-se ao início da vida sexual, ${ }^{7}$ visando a prevenção das IST e da gravidez na adolescência, causa de elevada morbilidade nesta faixa etária, e promovendo uma sexualidade livre, isenta de qualquer forma de violência e afetivamente enriquecedora. .,9,21 $\mathrm{O}$ aconselhamento contracetivo deve ser realizado de forma integral e esclarecedora de falsos conceitos, promovendo a responsabilização do/a adolescente no momento da escolha contracetiva. ${ }^{9,16,22}$

Para fornecer orientações aos profissionais e uniformizar práticas, surgiram recomendações internacionais e nacionais específicas para a faixa etária da adolescência. ${ }^{8,14,15}$ Mas há ainda alguma relutância na prescrição de determinados métodos contracetivos, que pode ser explicada pelo seu desconhecimento. ${ }^{10,23}$

Neste estudo, os clínicos mais novos (< 55 anos, $<20$ anos de carreira), do género feminino e os ginecologistas revelaram-se mais familiarizados com o aconselhamento contracetivo, em concordância com outros estudos internacionais, ${ }^{10,23}$ adequando a sua prática clínica às recomendações vigentes.

Apesar de globalmente os clínicos do género feminino estarem mais instruídos nesta temática, parece haver alguma insegurança no que respeita ao implante contracetivo. De acordo com as recomendações, o implante é ideal para adolescentes que prefiram um método com elevada durabilidade, que não requeira uma toma regular ${ }^{8}$ e que aceitem a amenorreia e/ou as hemorragias irregulares. ${ }^{16}$

Em função do grupo etário, os clínicos com pelo menos 55 anos (G4) referiram com maior frequência conhecer as recomendações nacionais e internacionais e sentem-se à vontade para abordar o aconselhamento contracetivo. Mas foram os clínicos com menos de 55 anos e com menos de 20 anos de carreira que revelaram maiores conhecimentos nesta temática, mostrando-se mais instruídos quanto à dispensa da realização do exame ginecológico, da citologia cervical e do estudo analítico, antes da prescrição de um método contracetivo.

Relativamente à especialidade, os ginecologistas revelaram-se mais familiarizados com o aconselhamento contracetivo, tal como o descrito na literatura. ${ }^{24}$ No caso particular dos métodos mais recentes, como o anel vaginal, o dispositivo intrauterino (DIU) e o sistema transdérmico, verificou-se igualmente maior conhecimento entre ginecologistas e clínicos com menos anos de carreira (A1 e IFEs), o que poderá explicar-se pelo investimento na formação/ formação mais recente. Verificou-se, no entanto, que os MGFs estão mais familiarizados com o implante contracetivo, o que poderá justificar-se por este ser um dos métodos disponíveis na consulta de planeamento familiar que, aliado à sua elevada durabilidade, se traduz num dos métodos de eleição das adolescentes, levando assim, a um maior conhecimento por parte de quem o aplica.

Apesar do DIU constituir uma opção segura e eficaz, apropriada para adolescentes ${ }^{8}$ vários estudos têm demostrado que este é pouco aconselhado por pediatras, ${ }^{8,11,25,26} \mathrm{O}$ que é concordante com os nossos resultados, provavelmente devido a um menor grau de familiaridade com o método, assim como à maior especificidade de conhecimentos exigidos para a sua aplicação. As razões apontadas nos estudos citados são a inexperiência na introdução do dispositivo, falta de capacidade de resposta ${ }^{8}$ ou falsa crença 
de que este método estaria associado a infertilidade futura. ${ }^{23}$ Concluem que a experiência com cada método e a sua forma de aplicação influenciam a sua recomendação. ${ }^{23}$

Os ginecologistas referiram mais frequentemente adequar o método contracetivo e a dose de estrogénio à adolescente. Entre os MGFs, o facto de os métodos que estão disponíveis na consulta de planeamento familiar para fornecimento à adolescente/jovem serem mais limitados, condicionando a prescrição dos clínicos, poderá justificar a menor frequência de adequação do método e da dose de estrogénios.

Globalmente, os ginecologistas sentem-se mais à vontade nesta temática, abordam mais as ISTs e revêm o método utilizado quando é solicitada a contraceção de emergência. Segundo as boas práticas, sempre que esta é solicitada, deve ter lugar a revisão do método utilizado e a educação à adolescente, para identificar possíveis erros de utilização ou falsos conceitos que limitem o seu uso apropriado. A contraceção de emergência deve constituir, portanto, uma oportunidade para uma contraceção eficaz. ${ }^{22}$

No que respeita ao direito à confidencialidade e privacidade do jovem, constata-se que os ginecologistas são os profissionais que mais frequentemente têm presente esse princípio na sua prática clínica, o que poderá advir do facto do aconselhamento contracetivo ser uma prática corrente a esta especialidade. De acordo com as recomendações da Sociedade Portuguesa de Medicina do Adolescente e da Sociedade Portuguesa de Contracepção, toda a informação deve ser prestada em ambiente de privacidade e confidencialidade, de forma clara e concisa, sem juízos de valor, abordando a sexualidade de uma forma integral e esclarecedora de falsos conceitos. ${ }^{16} \mathrm{O}$ adolescente deverá escolher livremente, de acordo com a sua necessidade, expectativa e condição médica, promovendo-se a sua responsabilização. ${ }^{16} \mathrm{~A}$ discussão contracetiva com os adolescentes é um dos fatores que motiva a instrução dos médicos acerca desta temática, uma vez que o conhecimento desses aspetos é fundamental para a sua prática clínica. ${ }^{25}$ Alguns estudos apontam inclusivamente o desconhecimento das recomendações vigentes, como uma das barreiras para o aconselhamento contracetivo. ${ }^{25}$

De acordo com as recomendações, a avaliação analítica, o exame ginecológico e a citologia cervical não devem ser realizados por rotina, exceto no caso do DIU, em que no dia da sua colocação, está previsto o rastreio de IST. ${ }^{16,27}$ A avaliação da tensão arterial, do peso e do índice de massa corporal (IMC) são os únicos parâmetros clínicos cuja avaliação é recomendada antes da disponibilização da maioria dos métodos. ${ }^{16}$

Neste estudo, foram os especialistas, nomeadamente os clínicos do grupo G2 (35 - 44 anos)/A2 (5 - 10 anos de carreira) e G3 (45 - 54 anos)/A3 (11 - 20 anos de carreira), aqueles que se revelaram mais instruídos nesse sentido. Por outro lado, os $\mathrm{P}$ são aqueles que menos frequentemente realizam o exame ginecológico pré contraceção, facto que poderá ser justificado pelo conhecimento de que este é dispensável na maioria das situações ou pela menor experiência na sua realização. Em contrapartida, os MGFs avaliam mais frequentemente o peso e o IMC, tal como preconizado.

De uma maneira geral, os $\mathrm{P}$ parecem estar menos familiarizados com o aconselhamento contracetivo, quando comparados com G e MGFs, tendo também a perceção de que os seus conhecimentos não estão atualizados, em concordância com o descrito na literatura. ${ }^{28}$ No entanto, é importante ressalvar que no caso particular dos $\mathrm{P}$, apenas os que se dedicam à área da medicina do adolescente estarão atualizados nesta temática, enquanto que os $\mathrm{G}$ e MGFs estão globalmente mais familiarizados, pois o aconselhamento contracetivo é parte integrante da sua prática corrente. Assim, os autores assumem que a menor taxa de respostas corretas observada no grupo dos $\mathrm{P}$ possa ser justificada por algum desconhecimento por parte dos respondentes não dedicados à área da medicina do adolescente, provavelmente mais desatualizados no aconselhamento contracetivo.

Conclui-se que os clínicos mais velhos (G4) e os G consideram estar atualizados no que toca ao aconselhamento contracetivo, sendo estes os que receberam formação recente na área, o que indicia que a formação tem um impacto positivo na perceção do nível de conhecimento. Destaque-se que os clínicos com menos anos de carreira e os $\mathrm{P}$ referem não se sentir devidamente instruídos na área e consideram necessária formação adicional. Apesar da perceção de que os conhecimentos estão atualizados, os IFEs também consideram pertinente a realização de mais formação.

Os resultados deste estudo indiciam que se deve apostar na formação dos profissionais, de forma a melhorar o aconselhamento contracetivo nesta faixa etária, que trará ganhos em saúde pública pela redução da morbilidade que acarreta. Um adequado aconselhamento contracetivo ao jovem/adolescente, constitui uma boa prática no âmbito dos cuidados antecipatórios, contribuindo para a diminuição de comportamentos sexuais de risco, assim como das comorbilidades a eles associadas, de que são exemplo as infeções sexualmente transmissíveis e a gravidez não desejada.

Apesar da concordância dos resultados com outros estudos, a técnica de amostragem (não aleatória) e o tamanho da amostra fragiliza a sua generalização. Sugere-se, portanto, replicar o estudo com uma amostra maior e mais representativa, no sentido de tentar compreender melhor as diferenças que, embora sem significado estatístico, este estudo indiciou.

\section{CONCLUSÃO}

À semelhança do descrito noutros estudos, ${ }^{23}$ pode concluir-se que o conhecimento acerca do aconselhamento contracetivo ainda não é ótimo. Constata-se que os clínicos do género feminino, os ginecologistas e os clínicos mais novos ( $<55$ anos e $<20$ anos de carreira) adequam mais frequentemente a sua prática clínica de acordo com as recomendações nacionais e internacionais, o que, no caso dos ginecologistas, pode ser atribuível à frequência de ações formativas recentes. 
Um correto aconselhamento sobre métodos contracetivos que responda às necessidades específicas de cada adolescente constitui um importante mecanismo de melhoria dos cuidados de saúde sexual e reprodutiva prestados, pelo que é fundamental investir na formação dos profissionais de saúde responsáveis pelo aconselhamento contracetivo.

Uma parte considerável deste trabalho relacionou-se com a construção e validação de um instrumento para colheita de dados sobre conhecimentos e práticas de contraceção em adolescentes de uma amostra de médicos em Portugal. Utilizou-se a Análise Fatorial Exploratória para analisar o padrão de correlações existentes entre as variáveis, usando esses padrões de correlações para agrupar as variáveis em fatores. Não obstante a apresentação e discussão dos resultados neste trabalho ter sido focado nas variáveis (itens) isolados, as características psicométricas das escalas são abonatórias à sua utilização em investigações futuras que permitam fazer uma análise por fatores.

\section{AGRADECIMENTOS}

À Sociedade Portuguesa de Ginecologia, em especial a Fernanda Águas e a Helena Leite, e à Associação Portuguesa de Medicina Geral e Familiar, em particular a Rui Nogueira, pela participação ativa no desenvolvimento deste projeto, nomeadamente no contributo na divulgação do questionário aos seus associados.

\section{REFERÊNCIAS}

1. Diário da República. Decreto-Lei n 259/2000. [consultado 2015 jan 27]. Disponível em: https://www.spdc.pt/files/legix/11256_3.pdf.

2. Mendes N, Palma F, Serrano F. Sexual and reproductive health of Portuguese adolescents. Int J Adolesc Med Health. 2014;26:3-12.

3. Ferreira M, Torgal M. Life styles in adolescence: sexual behaviour of Portuguese adolescents. Rev Esc Enferm USP. 2011;45:588-94.

4. Silva H, Ferreira S, Águeda S, Almeida A, Lopes A, Pinto F. Sexualidade e risco de gravidez na adolescência: desafios de uma nova realidade pediátrica. Acta Pediatr Port. 2012;43:8-15.

5. Matos M, Simões C, Camacho I, Reis M, Equipa Aventura Social. A saúde dos adolescentes portugueses em tempos de recessão. Lisboa: Aventura Social; 2015.

6. Miranda P, Aquino J, Monteiro R, Dixe MA, Luz A, Moleiro P. Comportamentos sexuais: estudo em jovens. Einstein. 2018;16:1-7.

7. Kaestle C, Halpern C, Miller W, Ford C. Young age at first sexual intercourse and sexually transmitted infections in adolescents and young adults. Am J Epidemiol. 2005;161:774-80.

8. Committee on adolescence. Contraception for adolescents. Pediatrics. 2014;134:642-49.

9. Coutinho C, Moleiro P. Aconselhamento sexual e contraceptivo aos adolescentes: a importância do gênero. Adolesc Saude. 2017;14:112-8.

10. Dehiendorf C, Levy K, Ruskin R, Steinauer J. Health care providers' knowledge about contraceptive evidence: a barrier to quality family planning care? Contraception. 2010;81:292-8.

11. Isaacs J, Creinin M. Miscommunication between healthcare providers and patients may result in unplanned pregnancies. Contraception. 2003;68:373-6.

12. Lee J, Parisi S, Akers A, Borrerro S, Schwarz E. The impact of contraceptive counseling in primary care on contraceptive use. J Gen Intern Med. 2011;26:731-6.

13. Kharbanda E, Stuck L, Molitor B, Nordin J. Missed opportunities for pregnancy prevention among insured adolescents. JAMA Pediatr. 2014;168:e142809.

14. Consenso sobre contracepção 2011. Documento conjunto das sociedades portuguesas de Ginecologia, Contracepção e Medicina da Reprodução. [consultado 2017 dez 6]. Disponível em: http://www.spdc.pt/ files/publicacoes/11_11363_2.pdf,

\section{PRÉMIOS OU APRESENTAÇÕES PRÉVIAS}

Trabalho apresentado sob a forma de comunicação oral no $18^{\circ}$ Congresso Nacional de Pediatria, vencedor do prémio Pfizer Vaccines - Prémio de Pediatria para estudos em Áreas diversas da Pediatria.

\section{PROTECÇÃO DE PESSOAS E ANIMAIS}

Os autores declaram que os procedimentos seguidos estavam de acordo com os regulamentos estabelecidos pelos responsáveis da Comissão de Investigação Clínica e Ética e de acordo com a Declaração de Helsínquia da Associação Médica Mundial.

\section{CONFIDENCIALIDADE DOS DADOS}

Os autores declaram ter seguido os protocolos do seu centro de trabalho acerca da publicação de dados.

\section{CONFLITO DE INTERESSES}

Os autores declaram não ter nenhum conflito de interesses na realização do presente artigo.

\section{FONTES DE FINANCIAMENTO}

Os autores declaram não ter recebido subsídios ou bolsas para a elaboração do artigo.

15. Hartman L, Monasterio E, Hwang L. Adolescent contraception: review and guidance for pediatric clinicians. Curr Probl Pediatr Adolesc Health Care. 2012;42:221-63

16. Neto S, Bombas T, Arriaga C, Almeida MC, Moleiro P. Contracepção na adolescência — recomendações para o aconselhamento contraceptivo. Acta Pediatr Port. 2014;45:51-63.

17. Hair JR, Black W, Babin B, Anderson, R, Tatham R. Análise Multivariada de Dados. Porto Alegre: Bookman; 2009.

18. Marôco J. Análise estatística com o PASW Statistics. Pêro Pinheiro: Report Number; 2010.

19. Cattell RB. The scree test for the number of factors. Multivariate Behav Res. 1966;1:245-76.

20. Streiner DL. Figuring out factors: the use and misuse of factor analysis Can J Psychiatry. 1994;39:135-40.

21. Gottschalk L, Ortayli N. Interventions to improve adolescents' contraceptive behaviors in low- and middle-income countries: a review of the evidence base. Contraception. 2014;90:211-25.

22. Committee on Adolescent Health Care. Counseling adolescents about contraception. Obstet Gynecol. 2017;130:e74-80.

23. Swanson KJ, Gossett DR, Fournier M. Pediatricians' beliefs and prescribing patterns of adolescent contraception: a provider survey. J Pediatr Adolesc Gynecol. 2013;26:340-5.

24. Schreiber C, Harwood B, Switzer G, Creinin M, Reeves M, Ness R. Training and attitudes about contraceptive management across primary care specialties: a survey of graduating residents Contraception. 2006;73:618-22.

25. Wilson SF, Strohsnitter W, Baecher-Lind L. Practices and perceptions among Pediatricians regarding adolescent contraception with emphasis on intrauterine contraception. J Pediatr Adolesc Gynecol. 2013;26:281-4

26. Papas B, Shaikh N, Watson K, Sucato G. Contraceptive counseling among pediatric primary care providers in Western Pennsylvania: a survey-based study. SAGE Open Med. 2017;5:1-8.

27. Klein D, Arnold J, Reese E. Provision of contraception: key recommendations from the CDC. Am Fam Physician. 2015;91:625-33.

28. Rubin S, Cohen H, Santelli J, McKee M. Counseling adolescents about the intrauterine contraceptive device: a comparison of primary care pediatricians with family physicians and obstetrician-gynecologists in the Bronx, NY. J Prim Care Community Health. 2015;6:162-9. 